\title{
UCSF Helen Diller Family Comprehensive Cancer Center
}

National Cancer Institute

\section{Source}

National Cancer Institute. UCSF Helen Diller Family Comprehensive Cancer Center. NCI

Thesaurus. Code C39532.

The UCSF Helen Diller Family Comprehensive Cancer Center has an overarching goal of shepherding new approaches to cancer prevention, detection, and treatment into clinical and population settings, where they can be tested and evaluated. The Center integrates the work of researchers and clinicians. It was designated as an $\mathrm{NCl}$ comprehensive cancer center in 1999. 